\title{
Larva migrans expérimentale chez un Primate, provoquée par un Ascaride de Serpent
}

\author{
Par Annie-J. PETTER, Odile BAIN \\ et L. ORCEL (pour l'étude anatomo-pathologique)
}

La majorité des cas de Larva migrans viscérale observés en clinique ont été attribués à l'Ascaris du Chien, Toxocara canis, et à celui du Chat, Toxocara mystax.

Cependant, Cl. Petter (1960), après avoir passé en revue la biologie des Ascaridiens, évoque la possibilité d'une infestation de l'Homme par d'autres Ascaridiens parasites d'animaux sauvages. Il existe en effet un certain nombre d'Ascaridiens dont le cycle comprend une migration larvaire dans un hôte intermédiaire vertébré à sang chaud et qui sont susceptibles, par l'écologie de leur hôte, d'entrer en contact avec l'Homme. En particulier, les Ascaridiens parasites de Serpents, qui présentent un cycle hétéroxène avec migration des larves dans de petits Rongeurs, seraient susceptibles d'être à l'origine de certains cas de Larva migrans viscérale.

Cette hypothèse de la possibilité d'une infestation de l'Homme par les larves d'Ascaridiens de Serpents a été reprise par Kutzer (1964), Sprent (1964), Kutzer et Grünberg (1964).

Afin de vérifier la possibilité du développement de ces larves chez les Primates, nous avons réalisé l'infestation expérimentale d'un Singe par des œufs d'Hexametra quadricornis (Wedl), espèce parasite de Vipères et de Cobras.

Le cycle de cette espèce a été étudié par Hörchner (1962); il a obtenu le développement des larves dans des Souris blanches; les larves du $2^{\circ}$ stade migrent dans le foie, puis passent dans la cavité générale où elles subissent la $2^{\circ}$ mue ; les larves du $3^{\circ}$ stade s'encapsulent dans le mésentère.

\section{Processus expérimental}

Dans le tube digestif d'une Vipère soufflante, Bitis arietans (Merrem), morte au Vivarium du Jardin des Plantes, nous avons recueilli un grand nombre d'Ascarides appartenant à l'espèce Hexametra quadricornis (Wedl).

Une vingtaine de femelles mûres ont été disséquées et les œufs mis en culture dans l'eau physiologique additionnée de bichromate de potassium. 
Ces œufs, examinés 12 jours plus tard, contenaient des larves du $2^{\circ}$ stade, enveloppées dans leur mue.

Le 10 juillet 1965, une grande quantité d'œufs sont donnés à ingérer à un Macacus cynomolgus $\mathrm{L}$.

Ce singe est sacrifié le 4 février 1966, c'est-à-dire sept mois plus tard. A l'autopsie, nous constatons que le foie est piqueté de nodules blanchâtres; de nombreux nodules de la même taille s'observent dans le péritoine; chacun de ces nodules contient une larve d'Ascaride enroulée en spirale ; à part une larve récoltée dans un caillot du cœur, les autres organes, poumons, cerveau, reins, sont indemnes; aucune larve libre n'est trouvée dans la cavité générale.

Les larves mesurent de $3 \mathrm{~mm}$ à $7,5 \mathrm{~mm}$; nous donnons les principales mensurations d'une larve trouvée dans une capsule du foie (fig. 1, A) ; longueur : 3,2 mm ; longueur de l'œsophage: $660 \mu$; anneau nerveux et pore excréteur situés à $250 \mu$ de l'extrémité antérieure; queue; $130 \mu$. Une vue de l'extrémité apicale montre une bouche triangulaire, 2 amphides et 4 grosses papilles submédianes; entre les papilles submédianes, on observe 2 reliefs médians (fig. 1, B).

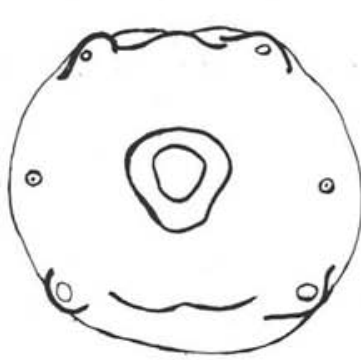

B

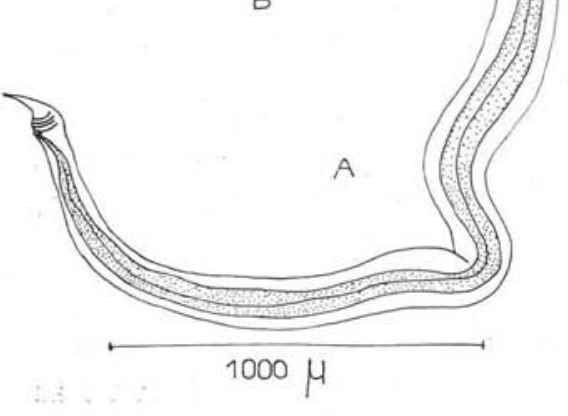

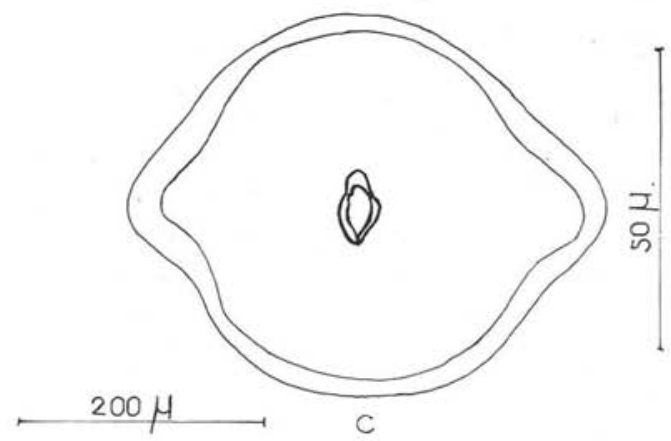
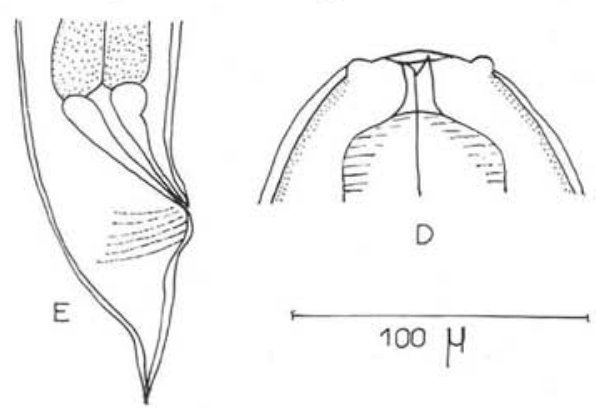

D

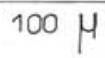

Hexametra quadricornis (larve trouvée dans une capsule du foie): A: corps entier, vue latérale ;

B: vue apicale superficielle; C: vue apicale profonde; D: extrémité antérieure, vue médiane; E:-extrémité postérieure, vue latérale 


\section{Etude anatomo-pathologique du foie}

Le parenchyme contient de nombreux granulomes plus ou moins organisés, partiellement développés autour des larves. Au point de vue topographique, ils sont disséminés dans les lobules hépatiques: certains siègent au contact ou au sein d'espaces portes; d'autres sont situés en plein lobule, parfois au voisinage de la veine sushépatique.

Ces granulomes sont d'âges différents: les plus jeunes sont, pour la plupart, dépourvus de larves, ils apparaissent formés par des amas histiocytaires infiltrés d'éléments inflammatoires polymorphes, avec fort pourcentage de polynucléaires éosinophiles. Le centre répond parfois à un petit foyer de nécrose éosinophile selon toute vraisemblance. Ces lésions jeunes répondent à la trace du cheminement du parasite dans le parenchyme et aux lésions qui lui ont succédé ; la présence de nécrose d'aspect fibrinoïde et d'une réaction inflammatoire assez riche en polynucléaires éosinophiles est en faveur d'un facteur allergique dans leur genèse.

D'autres granulomes, plus développés, réalisent progressivement un enkystement fibreux des larves. Il persiste encore une infiltration inflammatoire polymorphe, mais une sclérose lamelleuse assez dense prédomine par endroits; au contact du ver, on identifie encore des vestiges de tissu nécrotique.

Les espaces portes sont plus ou moins épaissis par la sclérose, avec une infiltration inflammatoire polymorphe variable, mais toujours assez riche en polynucléaires éosinophiles. Les phénomènes d'épaississement portal et périportal prédominent évidemment dans les espaces portes parasités. Quelques tractus scléreux intertrabéculaires ont tendance à unir certains espaces portes, mais il n'y a pas de lésions cirrhogènes.

La trame réticulinique est conservée ; elle est simplement épaissie vers les localisations parasitaires.

Il n'est pas possible de préciser si, au cours de leur cheminement, les larves empruntent préférentiellement les espaces portes ou des trajets veineux.

Enfin, il existe une stéatose microvésiculaire diffuse, touchant au moins $70 \%$ des hépatocytes.

En résumé, l'analyse des lésions constatées montre que les parasites cheminent dans le parenchyme en déterminant une réaction granulomateuse dans laquelle des facteurs allergiques locaux doivent intervenir; dans un second temps, ces parasites se fixent et s'enkystent; là, la sclérose prédomine et, au stade ultime, on observe même des petits nodules fibro-hyalins au sein desquels toute structure parasitaire a disparu.

\section{Conclusion}

Les larves d'Hexametra quadricornis sont donc capables de se développer chez les Singes; leur développement semble d'ailleurs chez ceux-ci être arrêté assez précocement en comparaison avec celui que l'on observe chez un Rongeur ; en effet, nous avons infesté avec des œufs provenant de la même culture un certain nombre de Praomys tullbergi, que nous avons sacrifiés à différentes dates; chez ceux-ci, dès le $30^{\circ}$ jour d'infestation, nous trouvions déjà, à côté de larves encapsulées dans le foie, des larves 
libres dans la cavité générale, dont les plus grandes atteignaient $1,5 \mathrm{~cm}$, alors que nous n'en trouvions aucune chez le Singe après sept mois d'infestation; après 110 jours d'infestation chez le Praomys, nous ne trouvions plus aucune larve dans le foie, mais de très nombreuses larves encapsulées dans la cavité générale, autour des reins et dans la paroi musculaire abdominale ; les plus grandes d'entre elles atteignaient 5,7 à $6 \mathrm{~cm}$.

Nous avons donc la preuve expérimentale que le développement des larves d'Ascaridiens parasites de Serpents est possible chez les Primates; ces larves, comme celles de Toxocara canis et Toxocara cati, s'encapsulent dans le foie, et sont donc susceptibles d'occasionner les mêmes troubles.

Etant donné que les Hexametra parasitent des Serpents très communs (Vipères et Couleuvres) qui fréquentent les potagers et peuvent souiller les légumes, il est très vraisemblable que certains cas de Larva migrans viscérale soient dûs à des larves de ces parasites.

\section{Bibliographie}

Kutzer (E.), 1964. - Die biologie einiger Schlangenaskariden. Proceed. VI. Int. Symp. Zootiererk. Wien.

— et Grünberg (W.), 1965. - Parasitologie und Pathologie der Spulwurmkrankheit der Schlangen. Zentralb. $f$. Veterinärmedizin, B, 12, 155-175.

HöRCHNER (F.), 1962. - Ein Beitrag zur Kenntnis des Entwicklungszyklus von Hexametra quadricornis Wedl 1862, Mozgovoy 1951 (Nematoda: Ascaridae). Z. f. Parasitenkunde, 21, 187-194.

Petter (C.), 1960. - Etude zoologique de la Larva migrans. Ann. Parasit. hum. comp., 35, 118-137.

SPRENT (J. F. A.), 1964. - Observations relating to the diagnosis of visceral larva migrans. Proceed. 1. Int. Parasitol. Congr. Rome.

[Laboratoire de Zoologie (Vers), Muséum National d'Histoire Naturelle, 57, rue Cuvier, Paris-5"] 